\title{
Identificación y solución de problemas para la participación ciudadana de los inmigrantes
}

\author{
Benno Herzog ${ }^{1}$ \\ Josefa Gómez-Moya \\ Esperanza Gómez-Guardeño \\ Juan Carlos Valderrama-Zurián \\ Rafael Aleixandre-Benavent \\ Universitat de València-CSIC \\ Insituto de Historia de la Ciencia y Documentación López Piñero \\ Palau de Cervero. Plaza Cisneros, 4. 46003 Valencia. \\ Benno.herzog@uv.es
}

\section{Resumen}

El objetivo del estudio es conocer las razones por las que los inmigrantes tienen un grado de participación política y social menor que los autóctonos que permita desarrollar medidas de inclusión de los mismos en el sistema democrático español.

Se entrevistaron en profundidad 26 inmigrantes de diferentes sexos, edades y países de origen sobre la relación existente entre los problemas personales y sociales con la búsqueda de estrategias de soluciones.

Los inmigrantes tienden a adoptar soluciones individuales para problemas estructurales, por lo que resulta necesario ampliar el modelo teórico de las estructuras de oportunidades políticas a un modelo que destaque la importancia de la percepción de las mismas. Palabras clave: inmigración, participación política, estructura de oportunidades políticas, investigación cualitativa.

\section{Abstract}

The aim of the study was to find out the reasons why immigrants tend to participate less than locals for developing an active policy of including newly-arriving immigrants in the Spanish democratic system.

An empirical study was undertaken in which 26 immigrants of different sexes, ages and countries of origin were given an in-depth interview about the relationship of personal and social problems with the search for solution strategies.

By broadening the theoretic political opportunity structure (POS) model towards a model of perceived political opportunity structure it was found that immigrants tended towards individual solutions for many structural problems.

Key words: immigration, political participation, political opportunity structure, qualitative research.

1. Benno Herzog ha podido participar en esta investigación gracias a la beca predoctoral V Segles, de la Universidad de Valencia. 


\section{Sumario}

$\begin{aligned} \text { Introducción } & \text { Conclusiones y discusión } \\ \text { Metodología } & \text { Agradecimientos } \\ \text { Resultados } & \text { Bibliografía }\end{aligned}$

Soluciones y participación ciudadana

\section{Introducción}

La participación social de la población en asuntos comunes es deseada no sólo desde el punto de vista de la ética democrática, sino también por motivos funcionales (Harwood y Myers, 2002; Heilmann, 2006), siendo ésta una de las principales razones por las que se han incrementado en los últimos años las investigaciones en esta área ${ }^{2}$.

Una de las líneas de investigación más destacadas es la que analiza la necesidad y la legitimidad de nuevos modelos de ciudadanía en sociedades multiculturales (Kymlicka, 1995; Benhabib, 2005; Chimni, 2001; Edwards, 2005; De Lucas, 2004). Estudios más empíricos se centran en la participación política a través de modelos estadísticos (Diehl y Urbahn, 1999), poniendo especial énfasis en la participación de inmigrantes a través de organizaciones «étnicas» (Jacobs y otros, 2004; Odmalm, 2004, Simó y otros, 2005) 3 $^{3}$ En estos estudios, se identificó una participación más baja de la población inmigrante que de la autóctona, lo cual se puede explicar tanto por los modelos del estatus socioeconómico (socio-economic-status (SES)) (Ramakrishnan y Espenshade, 2001), como por los modelos culturales de los países de origen (Bueker, 2005). Además, con la diferenciación de capital social bridging y bonding de Putnam (2000), se abrió el paso a una serie de trabajos que investigaban el asociacionismo como facilitador de la integración en la sociedad mayoritaria (Jacobs y otros, 2004; Odmalm, 2004) o como creador de comunidades aisladas, lo que Bauman (2000) llamó multicomunitarismo.

No obstante, la mayoría de los estudios empíricos realizados no explican las razones de la baja participación y no estudian a la gran mayoría de la población inmigrante que no participa. Se pueden formar cinco grandes conceptos teóricos que explican la aparición de participación social y política y su éxito como movimiento social (Koopmans, 1998): a) estudios como la obra clásica de Parsons (1959), que interpretan la implicación social y política como resultado de tensiones socioestructurales y, por lo tanto, son capaces de explicar procesos de cambios históricos; $b$ ) estudios basados en los conceptos del framing ('enmar-

2. Por ejemplo, el Journal of Ethnic and Migration Studies dedicó un número entero a este tema en mayo de 2004 y, en España, los Cuadernos electrónicos de filosofia de derecho giraban, en sus números 10/2004 y 12/2005, alrededor del tema.

3. En esta línea se enmarcan también los proyectos europeos POLITIS (Cyrus y otros, 2005) y Multicultural Democracy in European Cities (Morales y otros, 2005). 
cado') de Goffman (1959), con una fuerte relevancia para la explicación de la movilización de consensos ideológicos (Snow y Benford, 1992); c) modelos de identidades colectivas (Melucci, 1992), que explican la participación en movimientos culturales e identitarios; d) modelos basados en la posibilidad de movilizar recursos (McCarthy y Mayer, 1977), que ayudan, sobre todo, a entender la participación y el éxito de organizaciones y los lobbies profesionales, y e) el modelo de las estructuras de oportunidades (Kitschelt, 1986; Krisi y otros, 1995), el enfoque más usado en los últimos años, capaz de explicar las múltiples actividades políticas y sociales a pequeña escala, y que tiene en cuenta la complicada relación entre estado, instituciones e individuos. Este enfoque ha sido modificado a las necesidades de espacios concretos (Nentwich, 1996) o ampliado para integrar aspectos culturales dentro del modelo (Giugni y Passy, 2004).

En los últimos años, se ha mostrado, además, la necesidad de superar la división entre biografía y política, y entre cambio individual y colectivo, de modo que se deben de integrar las perspectivas biográficas en la investigación sobre participación política (Miethe y Roth, 2000).

Los inmigrantes en España no tienen los mismos derechos de participación que los autóctonos. Con la excepción de los inmigrantes comunitarios en las elecciones municipales, el derecho de voto se limita a los españoles. Otras formas de participación, como el derecho de manifestación y reunión, sindicación, huelga y asociación, quedaban restringidos a los inmigrantes «con papeles», una limitación que, en noviembre de 2007, fue declarada inconstitucional ${ }^{4}$.

La situación demográfica en España está caracterizada por el rápido crecimiento de la población inmigrante. Así, en el año 2000, el padrón municipal tenía registrados 923.879 extranjeros, lo que suponía un 2,28\% del total de la población (INE, 2000). Más de una tercera parte de estos extranjeros eran inmigrantes jubilados de los países del norte de Europa (International Retirement Inmigration (IRM)) (Casado-Díaz y otros, 2004; Casado-Díaz, 2006; Gustafson, 2001), cuyos grupos más numerosos estaban constituidos por los ciudadanos de Gran Bretaña y de Alemania, que residen sobre todo en las regiones mediterráneas. En 2007, la población extranjera se ha multiplicado por cuatro y hay 4.482 .568 extranjeros, lo que supone el 9,99\% del total de la población empadronada (INE, 2007). De ellos, el 12,86\% proviene de Marruecos (576.344 personas); el 11,72\%, de Rumania 5 (524.995), y el 9,4\%, de Ecuador (421.384). En su mayoría son buscadores de oportunidades laborales y aproximadamente el $30 \%$ de los inmigrantes extracomunitarios no tienen los papeles en regla.

4. Sentencia 236/2007, de 7 de noviembre de 2007. Recurso de inconstitucionalidad 17072001. Interpuesto por el Parlamento de Navarra contra diversos preceptos de la Ley Orgánica 8/2000, de 22 de diciembre, de reforma de la Ley Orgánica 4/2000, de 11 de enero, sobre derechos y libertades de los extranjeros en España y su integración social.

5. Se estima que, con la entrada en la Unión Europea de Rumania el 1 de enero de 2007, se ha duplicado la cantidad de residentes rumanos en España hasta el final del año. 
El número de inmigrantes en búsqueda de trabajo es mayor en los grandes municipios, es en estos centros urbanos donde se pueden experimentar, por primera vez, nuevas formas de convivencia y participación (Cucó, 2004).

El presente trabajo se centra en como perciben los inmigrantes la estructura social. El objetivo principal del estudio es conocer la relación entre trayectoria migratoria, problemas sociales y estrategias de solución de los mismos, con especial atención a la participación ciudadana desde el punto de vista de los inmigrantes.

\section{Metodología}

El estudio se basa en un análisis de 26 entrevistas semidirigidas con inmigrantes extracomunitarios (EU-25), 17 mujeres y 9 hombres de ocho nacionalidades distintas. Se incluyeron en la muestra también dos entrevistas con inmigrantes no extranjeros, es decir, personas que tenían una trayectoria inmigratoria pero que ya habían conseguido la nacionalidad española (tabla 1). Los entrevistados tenían una edad de 20 a 50 años y pertenecían a diversos estatus socioeconómicos y barrios de Alicante, Castellón y Valencia. Las entrevistas tenían una duración media de 45 minutos.

Los sujetos eran capturados en la calle o bien seleccionados a través de una base de datos que se generó en primavera de 2006, durante un estudio cuantitativo sobre participación de inmigrantes y en el que se les solicitó sus datos de contacto para estudios posteriores. Varios sujetos se negaron a participar en el estudio, porque consideraban muy delicado tratar el tema de la participación política, probablemente debido a que estaba planteado por investigadores de la sociedad mayoritaria (Beck-Gernsheim, 2004). En un caso se pidió parar la grabación durante la entrevista, para poder hablar de forma más abierta sobre el pasado político de la persona entrevistada.

Las entrevistas se realizaron, según las preferencias de los entrevistados, en una sala de la Universitat de València o en un ambiente más informal (parque o cafetería tranquila). Antes de realizar la entrevista, se agradecía a los participantes su colaboración en el proyecto y se subrayaba la importancia del estudio. Se les aseguraba el anonimato, se les informaba de que la duración media de la entrevista sería aproximadamente de una hora y de que serían gratificados por su tiempo y esfuerzo con un obsequio (tarjeta de regalo) por un valor de 15 euros. Posteriormente a esa introducción, existía la posibilidad de hacer preguntas y de aclarar dudas. Después, y tras el consentimiento de los sujetos, se iniciaba la entrevista según el guión previamente establecido. Todas las entrevistas se grabaron y se transcribieron íntegramente.

El tema central de las entrevistas era la relación con la sociedad de acogida y la participación ciudadana. Antes de abordar la participación ciudadana, era importante conocer a los interlocutores, por lo que se les preguntaba primero por puntos cruciales de su trayectoria de inmigración: el tiempo que llevaban en España, las razones por las que se habían ido de su país y habían venido a España, los problemas principales con los que se habían encontrado aquí 
Tabla 1. Entrevistas con inmigrantes según número asignado, fecha de la entrevista, ciudad, nacionalidad, género y edad

\begin{tabular}{llllll}
\hline No & Fecha & Ciudad & Nacionalidad & Género & Edad \\
\hline 1 & $26-05-06$ & Castellón & Ecuatoriana & Femenino & 22 \\
2 & $16-06-06$ & Valencia & Ecuatoriana & Masculino & 40 \\
3 & $28-04-06$ & Alicante & Colombiana & Masculino & 34 \\
4 & $29-04-06$ & Alicante & Rumana & Femenino & 23 \\
5 & $02-06-06$ & Castellón & Rumana & Femenino & 28 \\
6 & $01-06-06$ & Valencia & Marroquí y Española & Femenino & 40 \\
7 & $27-04-06$ & Alicante & Marroquí & Femenino & 20 \\
8 & $26-05-06$ & Castellón & Rumana & Femenino & 43 \\
9 & $20-04-06$ & Valencia & Argentina & Femenino & 24 \\
10 & $14-06-06$ & Valencia & Dominicana y Española & Masculino & 24 \\
11 & $25-04-06$ & Valencia & Colombiana & Masculino & 37 \\
12 & $28-04-06$ & Alicante & Boliviana & Femenino & 32 \\
13 & $25-09-06$ & Valencia & Argelina & Masculino & 43 \\
14 & $12-09-06$ & Valencia & Marroquí & Masculino & 35 \\
15 & $14-09-06$ & Valencia & Bulgara & Masculino & 32 \\
16 & $30-04-06$ & Alicante & Colombiana & Femenino & 44 \\
17 & $26-05-06$ & Castellón & Rumana & Femenino & 33 \\
18 & $26-05-06$ & Castellón & Rumana & Femenino & 50 \\
19 & $16-06-06$ & Valencia & Ecuatoriana & Femenino & 38 \\
20 & $14-09-06$ & Valencia & Colombiana & Femenino & 50 \\
21 & $19-09-06$ & Valencia & Rumana & Masculino & 39 \\
22 & $25-09-06$ & Valencia & Marroquí & Masculino & 20 \\
23 & $19-04-06$ & Valencia & Ecuatoriana & Femenino & 28 \\
24 & $29-04-06$ & Valencia & Ecuatoriana & Femenino & 44 \\
25 & $20-04-06$ & Valencia & Boliviana & Femenino & 40 \\
26 & $18-04-06$ & Valencia & Rumana & Femenino & 39 \\
\hline
\end{tabular}

y su relación con la sociedad de acogida. Posteriormente, se centraba la entrevista en los problemas tanto familiares, como del colectivo de los inmigrantes y de la sociedad de acogida en general. Finalmente, se les pedía que sugiriesen soluciones a todos estos problemas y, si no surgía ningún discurso sobre la participación política, se les preguntaba de forma directa sobre este tema.

Para el análisis, se tuvo en consideración la trayectoria migratoria de los inmigrantes, extrayéndose los puntos que posteriormente resultaban de interés para la definición de problemas o participación. Las entrevistas eran codificadas según las líneas principales del guión de entrevista. Se prestó especial atención al surgimiento tanto de recursos personales y estructurales, como de obstáculos en el análisis del comportamiento real a través de la red de oportunidades (Nentwich, 1996; Krisi y otros, 1992). Las oportunidades no se 
deben de entender como estáticas, sino como flexibles, en el sentido de que se puede obtener recursos que hasta el momento faltaban, pero también dependen en gran medida de la percepción de las oportunidades. Por lo tanto, se analizó también el cambio en la percepción de la estructura de oportunidades durante la estancia del inmigrante entrevistado en España.

Aunque se recogieron 26 entrevistas, el análisis se finalizó después de 18 entrevistas por saturación de información.

\section{Resultados}

\section{Trayectoria migratoria}

Todas las trayectorias de inmigración tenían en común que los inmigrantes pretendían mejorar su vida y tener acceso a recursos de los cuales no podían disponer en sus países de origen. En la mayoría de los casos, esos recursos se traducen en recursos económicos y trabajo. Además, la selección de España como país de destino no era casual y casi siempre se explicaba por las ventajas estructurales que mostraba frente a otros países. Pero, a partir de ahí, se reconocen grandes diferencias en las formas de inmigrar.

En la mayoría de los casos, es sólo una persona sin su familia nuclear la que viene a la Comunidad Valenciana. Esas personas intentan, en primera línea, mejorar su situación económica para, posteriormente, regresar a sus países de origen o traer a su familia. La decisión de inmigrar a la Comunidad Valenciana normalmente responde a que algún conocido o familiar lejano ya se encuentra aquí y es el primer punto de referencia.

También existen trayectorias, sobre todo de mujeres, que incluyen un cierto anonimato dentro de la nueva sociedad. Por lo que se refiere a este caso, se encuentran dos ejemplos típicos distintos. El primero es el de mujeres que vienen a ejercer la prostitución o caen en ella, por lo menos durante una temporada, lo cual significa, en general, una cierta ruptura con el entorno social principal, motivada por la vergüenza que padecen. El segundo ejemplo es el de mujeres que huyen de una situación familiar opresiva en búsqueda de más libertad en un país europeo, lo que conlleva, casi siempre, una ruptura con la familia de los países de origen, tal y como se puede ver en la siguiente cita de una mujer marroquí:

Pues porque, porque he tenido una, una mala experiencia y me casé ahí en Marruecos y no lo conocía ni nada, fue, pues fue por, no por la familia, digo vamos, porque yo lo acepté y mi madre me preguntó si quieres casarte o no, yyy, le dije que sí porque ella también me, me presionó un poquito pero que quieres quedarte la solterona del barrio, y claro allí no, no hay tanto trabajo como aquí una mujer puede, puedes encontrar trabajo, allí es un poquitín difícil, y, y me casé y nada, y, y mal, fue de lo mal, que no, no nos entendemos, no nos entendíamos ni nada, y a los dos meses pues me separé y cuando me, me, me divorcié, decidí no quedarme allí, porque la divorciada allí pues vale como lo que vale un, un pepino aquí, sabes, y vine aquí. Y ya. (Inmigrante 6) 
Dentro de un modelo muy simplificado, se puede hablar, por lo tanto, de factores que empujan (push), es decir, que hacen que una persona deje su país de origen, y factores que tiran (pull), responsables del hecho de que las personas vengan justamente a España. Éstos últimos se pueden describir muchas veces como redes sociales transnacionales ya existentes. Habitualmente se trata de una combinación de ambos y también de una cierta combinación de casualidades:

Eehh, en un principio yo eh, Colombiano, yo tenía un buen empleo en mi país, yy, pues, yo decidí porque pues allí hay, una serie de leyes que los trabajos, nooo, pues no, no duran mucho que digamos, entonces, pues, uuumm, al ver, en vista de que, me podría quedar sin trabajo allí dentro de mi país, pues decidí buscar un futuro por fuera, un futuro, y pues, en un principio que, quise ir a Estados Unidos, no se, me dieron las cosas, y, pues, decidí venirme aquí a España. (Inmigrante 11)

En este caso, se trata de falta de perspectiva laboral (push) y la expectativa de que en otros estados esto sería diferente ( $p$ ull), junto con la casualidad de que la primera elección de la persona no parecía posible.

Las diferencias en las razones para la inmigración pueden ser consideradas como cruciales para el seguimiento de la trayectoria migratoria, ya que la familia cumple un amplio abanico de funciones sociales sin las cuales la vida en la Comunidad Valenciana y el proceso de integración resultan mucho más difíciles. La familia aporta una solidaridad casi incondicional y ayuda en todo tipo de problemas. La familia normalmente tiene un papel fundamental, tanto ayudando a resolver problemas económicos, como en la ayuda social o el apoyo moral y psicológico, pero también en la estructuración del tiempo libre y del ocio. La ausencia de esta fuente de apoyo puede agravar cualquier situación de crisis y necesidad y aumentar el grado de marginación social.

Además de la familia, también existen diferencias de educación y de clase social entre los inmigrantes. Dichas diferencias tienen una implicación directa para el proceso de integración y también para la participación social. Entre los inmigrantes, se encuentran personas sin formación formal alguna y otros altamente formados, con carreras universitarias acabadas, personas que tienen un cierto nivel económico en su país de origen y gente que viene prácticamente sin nada. Pero la mayoría de las personas tiene en su país un nivel económico y una educación media-alta. Para empezar una nueva vida en otro país, hace falta tener, al principio, una cierta cantidad de dinero, sobre todo para emprender el viaje, y una cierta capacidad intelectual para afrontar una situación cultural nueva.

También el idioma es importante para los procesos de integración. Hay inmigrantes de países de habla castellana, lo cual facilita enormemente la comunicación, y personas que primero tienen que aprender el idioma. Aquí también influyen factores como la cercanía de la lengua original al castellano (per- 
sonas procedentes de Rumanía suelen tener más facilidad en aprender el castellano) y el nivel de estudios.

Desde el enfoque de género, existen diferencias relevantes en las posibilidades de ascenso social. Las mujeres suelen ser más vulnerables socialmente y están peor pagadas que los hombres. Asimismo, suelen tener la mayor responsabilidad en la familia y son víctimas de violencia y de explotación sexual con mayor frecuencia que los hombres.

También la edad con la que una persona inmigra tiene efectos directos para las perspectivas de integración. Hay personas que inmigran con sus padres cuando todavía son niños, algunos incluso pasan una temporada relativamente larga de formación en España y otros vienen ya en edades más avanzadas directamente a la búsqueda de trabajo.

Finalmente, las personas recién llegadas suelen estar menos integradas que las que ya viven aquí durante muchos años, y en la escala del ascenso social suelen estar más abajo.

Todas estas diferencias hay que tenerlas en cuenta cuando se habla de «los inmigrantes» o de «los problemas» en general. Además, los efectos descritos suelen ser acumulativos, es decir, una mujer sin familia ni recursos económicos, con pocos estudios y de un país de habla árabe suele tener más problemas que un hombre, licenciado, castellanoparlante y que sigue a su familia. También será difícil, justamente por esas diferencias, hablar de un solo discurso, ya que las diferentes tradiciones participativas, niveles educativos, grados de integración, etc. no producen, probablemente, una forma de participación homogénea.

\section{Principales problemas}

Durante las entrevistas, surgieron una gran cantidad de problemas actuales y pasados: de carácter individual, de todo el colectivo de inmigrantes o de la sociedad española en general ${ }^{6}$. Algunos problemas se han podido solucionar, o por lo menos se ha podido quitar la urgencia con la que se presentaban. Como nota metodológica, se observa que, con el paso de los años y la información de que actualmente dispone el inmigrante, puede cambiar la percepción de los problemas pasados.

Se puede decir que una gran parte de los problemas con los que los inmigrantes se encuentran, sobre todo al principio de su estancia, en España son problemas estructurales y relacionados con el estatus legal. Pero la situación de ilegalidad con la que la mayoría de los inmigrantes se encuentran en un momento dado y el largo proceso de regularización constituyen un importante problema, porque conlleva un gran número de dificultades relacionadas con ella.

6. Para un estudio más exhaustivo sobre los principales problemas de la población inmigrante en la Comunidad Valenciana, véase Ceim, 2004. 


\section{El trabajo}

Sin papeles, la posibilidad de trabajar y de ganar dinero, es decir, la principal esperanza por la que viene la mayoría de los inmigrantes, está fuertemente restringida. No se puede trabajar con contrato, y las personas «ilegales» se ven forzadas a trabajar en economía sumergida, por un bajo sueldo y con pocos derechos. Además, muchos inmigrantes se quejan de que no existe la misma disposición de pagar a un inmigrante que a un autóctono. Se percibe con total naturalidad que se exige a los inmigrantes trabajar igual que los autóctonos pero por menos sueldo.

[...] sobre lo que, como me ha ido, pues la verdad, yo que sé, es, es duro un poquito. Es un poquito duro, sobre todo cuando no tienes papeles, que ves que no puedes, claro, no puedes encontrar trabajo. Y si lo encuentras, se aprovechan de ti, y, ya está. Económicamente, pues, no tan bien como pensaba yo, porque esto de [...] lo que te he dicho, yo mi marido lo conocí y no tenía ni un duro, o sea que, no vine con la idea esa de, de, de buscar un hombre rico, ni nada, o sea, él, dejó de, dejó, se le acabó el contrato del bar, y lo dejó. Y, desde ahí pues empezó a buscar trabajo como cualquiera, en empresas temporales, ahora trabaja un mes ahora está en el paro y, yo pues estaba trabajando en una casa, y me pagaban cuarenta mil pesetas desde las nueve de, de la mañana hasta las tres, tres y media, y, y te duele mucho porque, te sientes que, sabes que se están aprovechando porque no tienes papeles, porque no sabes hablar, y mucho, una cosa que yo no la haría, ¿sabes? (Inmigrante 6)

También existe el problema del reconocimiento de sus titulaciones extranjeras para poder acceder a trabajos más cualificados. Este proceso, que tarda mucho y parece poco transparente, defrauda directamente la esperanza de que con la formación recibida en su país de origen puedan aspirar a buenos trabajos en España. Este problema se relaciona a veces de forma indirecta con un cierto racismo por parte de la sociedad de acogida.

Otro problema relacionado con el trabajo es la forma en la que se suele encontrar trabajo: de boca a boca y a través de contactos, lo que puede ser un obstáculo para personas con pocos contactos. Además, la mayoría de los contactos que tienen son con personas en la misma situación y no con personas que, a través de su alto capital social, puedan facilitar el acceso a puestos de trabajo.

Sea cual sea la razón, el resultado es que muchos inmigrantes al principio ven defraudadas las esperanzas con las que vinieron a España.

\section{La vivienda}

El acceso a una vivienda digna también se ve restringido por la población inmigrante y está vinculado con lo dicho anteriormente. El bajo sueldo fuerza a compartir piso y a vivir en viviendas en mal estado. Además, muchos dueños de pisos exigen la existencia de un contrato laboral para poder alquilar el piso, condición que no todos pueden cumplir. Como problema añadido, muchos propietarios no alquilan viviendas a personas inmigrantes, lo cual se percibe como racismo por parte de los inmigrantes. 


\section{La familia}

La posibilidad de vivir con la familia también está relacionada con los problemas descritos. Estatus legal, trabajo (dinero) y el acceso a la vivienda condicionan la posibilidad de reunir a la familia.

Para muchos inmigrantes, la ausencia de la familia se convierte en problema. Si bien la familia es el lugar de protección de conflictos exteriores, es ahí donde también se muestran una gran cantidad de conflictos. No poder cumplir con el papel deseado, cambios en el poder familiar, choques culturales y con ello intergeneracionales, pueden hacer surgir problemas familiares. Pero independientemente de la presión exterior, surgen también dentro de la familia conflictos intergeneracionales normales, pero con la diferencia de que, además, muchas veces padres e hijos parecen pertenecer a culturas diferentes.

\section{Problemas psicológicos}

Las situaciones descritas ejercen una presión enorme sobre las personas, que sienten defraudadas parte de sus esperanzas. Esto ocasiona problemas psicológicos que muchos inmigrantes padecen y que suelen ir desde la tristeza hasta la depresión.

\section{Racismo}

En muchas entrevistas, surgió el tema del racismo que sufren los inmigrantes por parte de algunos miembros de la sociedad de acogida. Éste se expresa más sutilmente en tratamientos menos favorables, sueldos más bajos, etc., pero a veces existe un racismo abierto, y tienen que soportar hasta insultos directos.

$\mathrm{Al}$ identificar este problema, algunos lo perciben como racismo, mientras otros lo perciben en términos distintos, como ignorancia o poca cultura.

\section{Problemas de sociabilidad}

El sentimiento de inferioridad que muchos inmigrantes relatan como consecuencia de su situación y del conjunto de los problemas mencionados, les convierten en temerosos a la hora de socializarse con otras personas. Muchas veces buscan protección con otras personas inmigrantes y las relaciones con los autóctonos fuera del entorno laboral son escasas.

\section{Idioma}

El idioma es un problema para los inmigrantes de países de habla no hispana, pero también se suele solucionar con el tiempo, ya que, tarde o temprano, casi todos aprenden el castellano.

El idioma permite integrarse en la sociedad de acogida, pero, al mismo tiempo, la integración favorece el aprendizaje del idioma o, dicho al revés, la separación dificulta el aprendizaje del idioma y, de este modo, obstaculiza la posibilidad de superar esa separación.

Los inmigrantes vienen a España con unas esperanzas enormes de mejorar su nivel de vida. Son esperanzas típicas de clases ascendentes que, con el tiem- 
po, con el trabajo, con la educación y con el paso de las generaciones, mejoran su situación social. Aunque a largo plazo estas esperanzas muchas veces se ven cumplidas, el principio de la gran mayoría de historias de migración es largo y duro, pues se encuentran con un trabajo mal pagado, en un estado que tarda mucho en reconocer su formación o no la reconoce del todo e incapaces de reunirse con su familia.

Aunque muchas veces estos problemas están percibidos como problemas personales, se muestra claramente que se trata de problemas estructurales, ya que casi todos los inmigrantes extracomunitarios pasan por una gran cantidad de problemas parecidos. Pero no todos los problemas son exclusivamente de inmigrantes. También hay algunos compartidos con muchas otras personas autóctonas, como la explotación laboral o la discriminación por género. Aunque ésos son problemas que padecen también algunos españoles, existe una situación de mayor vulnerabilidad por parte de los inmigrantes, es decir, tienen menos recursos para defenderse en contra de situaciones injustas.

En las entrevistas, se hablaba también de problemas sociales en general, tal y como se discuten a diario en los medios de comunicación. La procedencia de los inmigrantes ofrecía, en este aspecto, tanto obstáculos como ventajas para la identificación de problemas sociales. Como la principal razón de la inmigración era que en España se vive mejor, algunos inmigrantes no percibían graves problemas económicos o políticos. En comparación con la situación de sus países de origen, la calidad de vida aquí les parece tan buena que no pueden formular críticas respecto a estos temas.

Pero la experiencia de haber vivido en dos culturas distintas les permite tener una perspectiva más amplia y valorar otras cosas. Se trata, sobre todo, de aspectos relacionados con el estilo social, la sorpresa de que el alto nivel de vida no conlleva una satisfacción rotunda de la población española, sino que existen problemas como la droga, el vandalismo, jóvenes aburridos, es decir, el hecho de que existan personas que han logrado aquello a lo que los inmigrantes aspiran y sin embargo no son felices. Lo muestra la siguiente cita sobre jóvenes que toman drogas porque se aburren:

[...] no tiene ningún sentido, y molesta, visto desde fuera, para quien ha venido, y sobre todo para quien viene y le gusta esto. Igual hay gente que igual tiene rechazo hacia aquí, y dice, a mi me da igual, que hagan lo que quieran. Pero cuando vives aquí y te gusta no sé, Valencia o España o lo que sea, y te sientes parte de esto, te da rabia, porque dices es que es un, es un sitio que realmente vale la pena, y esa gente es como que no lo merece, ¿sabes?, no sé, te da rabia, te da mucha rabia, y dices, yo estoy como luchando día a día para que me quieran, y digan que eres uno más, y ellos que lo son, de repente es como no me importa nada mi vida, voy a meterme cualquier cosa y me voy a quedar seco por ahí, es ridículo, completamente ridículo. (Inmigrante 10)

Es obvio que no todos los inmigrantes padecen todos los problemas, aunque se trate de problemas relativamente comunes. Algunos cuentan que han tenido suerte, que han podido legalizar su situación muy rápidamente, que 
encontraron un buen trabajo en seguida o que tienen relaciones familiares con españoles, lo que les ha facilitado la integración. No obstante, los problemas muchas veces están conectados, por ejemplo a través del estatus legal. Además, existe una tendencia hacia la acumulación de problemas y así, y por falta de recursos, hacia una mayor vulnerabilidad de los inmigrantes.

A veces, en la definición de problemas sociales, se observan tendencias excluyentes, elitistas y separatistas. La definición como problemática y conflictiva de algunos grupos de inmigrantes, a los que uno mismo no pertenece, puede ser augurio de futuros conflictos entre los inmigrantes.

\section{Soluciones y participación ciudadana}

La historia de migración, la situación social y la identificación de problemas conduce a estrategias de soluciones de problemas específicos. Son soluciones basadas en el propio esfuerzo individual, soluciones colectivas y soluciones desde la política «de arriba». Sólo algunas de ellas se pueden considerar participación ciudadana.

Tal como se observa en otros estudios (Diehl y Urbahn, 1999), la participación de la población inmigrante está por debajo del nivel de participación de la población autóctona. Las razones para tal abstinencia participativa son:

a) Soluciones individuales y familiares

La identificación de muchos problemas estructurales como problemas individuales condiciona la búsqueda de estrategias de solución, como muestra el siguiente ejemplo:

No sé, es que no, no pienso en soluciones, porque ya pienso en solucionar mi propia vida, en solucionar problemas de estos grandes, ya, no, no tengo tiempo para pensar en estas cosas. (Inmigrante 16)

Bajo «solución» se entiende aquí no un cambio político o estructural, sino cambiar la propia posición individual dentro de la sociedad.

Como se trata de problemas individuales, se apunta a soluciones individuales: el ascenso personal mediante esfuerzo y tiempo, y la mejora de vida a lo largo de las generaciones:

[...] que ella [la hija de la interlocutora] se siente con la tranquilidad de que estamos en un lugar donde vamos a tener nuevas oportunidades y que está en nosotros que nosotros podamos salir adelante y que podamos prosperar de diferente forma. (Inmigrante 1)

Sobre todo el estrés psicológico se percibe como algo que simplemente tiene que pasar con el tiempo. En este sentido, la búsqueda de soluciones puede apuntar a la mejora de la situación personal en España o a volver al país de origen. 
Se trata, por lo tanto, de gente que busca soluciones personales para problemas estructurales, lo cual también se puede contemplar en el mero hecho de haber inmigrado. De los problemas estructurales de sus países de origen, de tipo económico, político y social se buscaba la solución individual (y, no obstante, compartida con muchos de sus compatriotas) de emigrar. Eso puede explicar en cierto grado la poca participación del colectivo inmigrante.

Aunque la búsqueda de soluciones muchas veces es individualizada, se desarrolla dentro de una configuración social. Sobre todo buscan redes sociales como ayuda para mejorar la vida personal. Estas redes sociales están formadas, por ejemplo, por la familia. Pero con la familia también se asocia otra forma de mejora: aumentar el nivel social a través de las generaciones.

La educación es el eje central para conseguir la mejora con el tiempo, y la escuela, la protagonista de esa esperanza, aunque también se reconoce la importancia de los padres en este proceso. A través de la escuela, los propios niños se integran, pero también los padres entran en contacto con otros padres e incluso se cambia un poco la sociedad hacia la tolerancia y la interculturalidad.

\section{b) Soluciones colectivas}

Las redes sociales de los inmigrantes no sólo consisten en la familia nuclear. Muchas veces, se trata de familia en un sentido muy extenso. Además, esas redes incluyen también conocidos y asociaciones de y para inmigrantes. Pero las asociaciones en primera línea, y sobre todo al principio, no se buscan para cambiar estructuras sociales, sino como recurso personal.

El conocimiento de este tipo de asociaciones predetermina también su visión de la participación social, en general, y del asociacionismo, en particular. Los inmigrantes conocen de su propia experiencia sobre todo a las asociaciones de ayuda social o de ayuda directa y no conocen la amplia gama de asociaciones reivindicativas. Por lo tanto, entienden el asociacionismo como un sistema de ayudas directas. Si los inmigrantes hablan sobre su propia participación en asociaciones, se encuentra este modelo de asociacionismo, el modelo de ayuda humanitaria poco reivindicativa:

Sí, me gusta mucho, me gusta, a mi me gustaría trabajar en, algo así Cruz Roja, o ayudante de, así, ir a, a casas de abuelitas que están solas y ayudar, pero claro, de momento con el niño pequeño no puedo, entonces yo, pagar de mi bolsillo lo que no tengo, e ir a ayudar a otros, pues primero atender los míos, ¿sabes? Pero sí que me encantaría, te lo juro, me encantaría ayudar a extranjeros o a españoles que necesitan ayuda, cualquier cosa. (Inmigrante 6)

Como muchos inmigrantes han pasado por varias asociaciones en busca de apoyo, consideran las asociaciones en general como algo positivo que ayuda a los demás, aunque a veces se quejan de su mal funcionamiento, de la insuficiencia o de intereses económicos dentro de ellas.

Entre las razones que daban los inmigrantes para la baja participación, están la falta de tiempo, la actitud de intolerancia por parte de la sociedad de acogida, 
si bien se trata de una falta de competencias interculturales, es decir, el sentimiento de los inmigrantes de sentirse excluidos, de ser mirados como «raros». Otros argumentos que surgían durante las entrevistas eran la propia incompetencia, la falta de formación o de conocimiento de la lengua y, en un caso, "la voluntad de Dios».

También destaca como motivo para la baja participación la falta de integración, la cual también depende de la participación. El primer paso para participar es el ser invitado por alguien a participar; sin embargo, la falta de contacto personal con españoles hace que los inmigrantes no se enteren de las posibilidades de participar y no se sientan invitados a formar parte de asociaciones.

\section{c) Soluciones «de arriba»}

La esperanza de que Dios arregle los problemas era una excepción dentro de las posibles soluciones «de arriba». Mucho más a menudo se esperaba de «los políticos»o "la política», en un sentido muy abstracto, soluciones para problemas sociales. Cuando se reflexiona sobre la influencia de la política en los problemas sociales, destacan varios aspectos:

- El desconocimiento del funcionamiento de la estructura democrática española. Esto tiene como resultado, respecto a la opinión sobre los agentes políticos, la creación de paralelismos y llanezas del tipo «todos son unos mafiosos», sin poder dar ejemplos concretos:

Ah, yo sacaría a, haría una limpieza de políticos, no dejaría ni uno. Tendría que renovarse todo. Ponerse gente que tuviese otra idea, otra cabeza. Gente joven que quiera a su país, que quiera a su país y a su gente. Lo que pasa es que eso cómo se hace si hay toda una mafia detrás, si hay mucha gente que no se conoce. (Inmigrante 9)

No obstante, también existe la esperanza que «ellos de arriba» arreglarán las cosas, esperanza que se ve justificada por el alto nivel de vida que a veces se interpreta como resultado directo de que los políticos «llevan bien el país». Ambas posiciones conducen a una baja participación, ya que, en el primer caso, no se puede hacer nada mientras, en el segundo, no hace falta hacer nada.

- El desconocimiento del funcionamiento de la estructura democrática española y del funcionamiento de democracias participativas, en general, también tiene otras consecuencias negativas para la participación de los inmigrantes. Primero, la participación muchas veces no está vista como pilar central de la democracia, sino como una molestia, como «meterse con alguien» y no como una práctica deseada también por la Administración:

Por ejemplo, en este momento estoy para recrearme un poco, no me quiero meter en problemas, ¿me entiendes? Creo que las manifestaciones y todo esto también es meterse en, en preocuparse, en que tienes que realmente estar luchando por algo. (Inmigrante 9) 
La segunda consecuencia del desconocimiento de las estructuras es la percepción de que no existe un vínculo entre el individuo y la Administración. Las ideas para cambiar situaciones sociales se vuelven, de ese modo, muy abstractas. Se pronuncian amplios discursos sobre lo que tienen que hacer «los políticos», pero no se percibe la influencia de grupos de presión y de los individuos en ese proceso. Al final, sólo queda para el individuo el camino poco participativo de trabajar duro, formarse y lograr, de ese modo, una mejora de su situación personal. Incluso muchos inmigrantes pronuncian sus deseos hacia la Administración en el sentido de mejorar la situación general a través de la mejora de la educación.

Por tanto, se perciben tres tipos de soluciones principales para los inmigrantes:

1) El cambio individual, familiar o generacional.

2) La ayuda humanitaria a través de asociaciones de ayuda directa.

3) El cambio a través de «los políticos».

Pero falta el conocimiento de cómo lograr que en la política se cambien las cosas y se perciben pocas relaciones entre la situación personal y la situación política. Como se ha visto, las situaciones personales tienen problemáticas estructurales comunes que muchas veces están percibidas como problemas personales, singulares. Para mejorar la situación de los demás, se conoce la ayuda humanitaria, entendida como dar algo de sus recursos a otras personas, lo cual resulta muy difícil para gente con pocos recursos. La idea de que, a través del asociacionismo, se percibe algo personalmente o que justamente el asociacionismo sirve como un vínculo de la sociedad civil hacia la Administración, sólo es percibida por personas que tienen un papel activo dentro de una asociación.

Aunque los inmigrantes representan un grupo menos participativo, también en las entrevistas surgían algunas formas de participar por parte ellos, sobre todo en asociaciones específicas de inmigrantes, pero también en asociaciones de la sociedad de acogida.

\section{Conclusiones y discusión}

En el presente estudio, se ha observado que existe una relación entre la situación personal de los inmigrantes, los problemas sociales que tienen y la participación ciudadana. Pero esta relación no está determinada ni es objetiva, sino que está mediada por las percepciones subjetivas de los inmigrantes. Es decir, depende de cómo se perciben los problemas y la propia situación dentro de la sociedad y el sistema político, se desarrollan estrategias de solución distintas.

Esa relación se manifiesta de tal modo que si los problemas son percibidos como personales, se tiende a buscar soluciones de modo individual, mientras que si se perciben como un problema social, se está más predispuesto a la participación ciudadana. No obstante, hay una fuerte tendencia a percibir pro- 
blemas colectivos y estructurales en términos individuales y, por lo tanto, a abogar por soluciones individuales. Muchos de los problemas sociales con los que se encuentra la población inmigrante están vinculados a la situación legal en la que se hallan y afecta a múltiples aspectos de su vida diaria. Asimismo, se observa un desconocimiento de las posibilidades de participación y una tendencia a considerar que su participación no es deseada y, "por no molestar", no participan.

Destaca también la relación entre integración y participación en ambos sentidos. La integración fomenta la participación tanto como la participación ciudadana fomenta, con la necesidad de crear vínculos con personas e instituciones de la sociedad de acogida, la integración (Schrover y Vermeulen, 2005) o, en otras palabras: la exclusión social dificulta una participación democrática del colectivo inmigrante. Koopmans (2004) argumenta, en este sentido, que la integración forma parte de una estructura de oportunidades políticas que incrementa la participación. La participación ciudadana puede ser una respuesta al sentimiento de pérdida de legitimidad del sistema político y de la cohesión social (De Lucas, 2004), tanto desde aspectos éticos y democráticos, como desde una perspectiva de funcionalidad, puesto que puede incrementar:

- La integración social y política. Estar en contacto con personas e instituciones de la sociedad de acogida mejora la integración de los inmigrantes y facilita a los autóctonos a aprender a convivir en la interculturalidad.

- La cohesión social. Al ser responsables, tanto los inmigrantes como los autóctonos, de asuntos comunes, se fomenta la creación de un sentimiento de pertenencia y una identidad compartida.

- La legitimación del sistema político. Incluir a un alto porcentaje de la población a participar en la democracia puede favorecer la disposición de aceptar decisiones políticas y disminuir el riesgo de comportamiento desviado por los colectivos excluidos.

Pero ese deseo a la participación puede encontrarse con obstáculos por parte de la población inmigrante, como la falta de recursos, tiempo, dinero, conocimiento de las estructuras democráticas, problemas lingüísticos y falta de derechos.

La necesidad objetiva, los obstáculos o recursos objetivos no determinan la activación social y política de los inmigrantes, sino que se ven mediados a través de la percepción que tienen de la situación social y de las posibilidades para cambiarla. Esto hace referencia a un modelo de estructura de oportunidad que se ha establecido en las ciencias políticas en los últimos años (Koopmans, 2004; Danese, 2001; Schrover y Vermeulen, 2005), en el sentido de que no se trata de oportunidades reales para la participación, sino de las posibilidades percibidas que influyen en la activación participativa de los inmigrantes. En el ámbito de los actores sociales, se puede hablar, por lo tanto, de un modelo de estructura de oportunidades politicas percibidas. 
Esto también explica lo que en un primer momento puede parecer una paradoja. Los que menos problemas tenían, más proactivos eran a la hora de participar. La ausencia de problemas que impiden la participación o, mejor dicho, la existencia de recursos que lo facilitan, aumentan la participación social.

Hasta el momento, existen pocos trabajos publicados sobre el fenómeno de participación de los inmigrantes y aún menos los que relacionan la participación con las trayectorias personales y las historias de vida de las personas inmigrantes. Investigaciones previos respecto a la situación social de los inmigrantes (Ceim, 2004), respecto a la participación social y política de los mismos (Diehl y Urbahn, 1999) o respecto a la relación entre la participación y la integración (Junn, 1999) suelen confirmar las observaciones del presente estudio. Existen diferentes modelos de explicación para tal hecho. La combinación de modelos de estatus socioeconómico con factores culturales puede explicar también las diferencias entre los diversos grupos de inmigrantes (Ramakrishnan y Espenshade, 2001; Bueker, 2005).

También la estructura de oportunidades como importante marco para la participación y la integración se confirma, entre otros, en el estudio de Daneses (2001). El estudio de Pardo (1990) subraya, además, la importancia de las amistades, las redes sociales y las redes étnicas, sobre todo para actividades grass-root. En especial, las mujeres inmigrantes transforman sus redes sociales para la movilización alternativa no institucionalizada o en recursos políticos.

En el futuro, debería tenerse en cuenta que la exclusión de gran parte de los inmigrantes extracomunitarios por su estado de ilegalidad puede provocar conflictos sociales a los que hay que anticiparse mediante la observación de los cambios que ocurran en la población inmigrante, las nuevas disposiciones legales y adaptando la política social a una nueva realidad que contemple la relación entre participación e integración.

\section{Agradecimientos}

Se agradece la colaboración de las trabajadoras de campo Leticia Álava Gutiérrez y Laura Paneque de la Torre. Este artículo se ha realizado utilizando los datos obtenidos de un proyecto financiado por la Dirección General de Participación Ciudadana de la Conselleria de Inmigración y Participación de la Generalitat Valenciana.

\section{Bibliografía}

Bauman, Z. (2000). Community. Seeking safety in an insecure world. Cambridge: Policy Press.

Beck-Gernsheim, E. (2004). Wir und die Anderen: Vom Blick der Deutschen auf Migranten und Minderheiten. Frankfurt/Men: Suhrkamp.

BeERLI, A.; DíAz, G. (2003). «La autoimagen y las motivaciones del voluntariado como factores explicativos del tipo de ONG en que se colabora». Esic Market: Revista Internacional de Economia y Empresa, enero-abril. 
BenHABIB, S. (2005). «On the alleged conflict between democracy and international law. Ethics \& International Affairs, 19 (1).

BUEKER, C. S. (2005). «Political incorporation among immigrants from ten areas of origin: The persistence of source country effects». International Migration Review, 39 (1).

CASADO-DÍAZ, M. A.; KAISER, C.; WARNES, A. M. (2004). «Northern European retired residents in nine southern European areas: characteristics, motivations and adjustment». Ageing and Society, 24 (3).

CASADO-DíAZ, M. A. (2006). «Retiring to Spain: An analysis of differences among North European nationals». Journal of Ethnic and Migration Studies, 32 (8).

CASTles, S.; Miller, M. J. (2003). The age of migration. Basingstoke: Palgrave Mcmillan.

Chimni, B. S. (2001). «Reforming the International Refugee Regime: A Dialogic Model». Journal of Refugee Studies, 14 (2).

Ceim - Centro de Estudios para la Integración Social y Formación de INMIGRANTES, FundACIÓN DE LA COMUNidAD VALENCIANA (ed.) (2004). Igual de seres humanos: Historias de inserción de migrantes con problemas en la Comunidad Valenciana. Valencia: CeiM D.L.

CiS, Centro De Investigaciones Sociológicas. (2005). Representación y participación política en España. Estudio CIS no 2588.

CuCó Giner, J. (2004). Antropología Urbana. Barcelona: Ariel.

Cyrus, N.; Gropas, R.; KosIC, A.; VOGEL, D. (2005). Opportunity structures for immigrants' active civic participation in the European Union: sharing comparative observations [en línea]. University of Oldenburg. POLITIS-Working paper No. 2. <http://www.uni-oldenburg.de/politis-europe/download/WP2 POLITIS_CyrusGropasKosicVogel_2005.pdf> [Consulta: 15 de abril de 2007]

DANESE, G. (2001). «Participation beyond citizenship: migrants' associations in Italy and Spain». Patterns of Prejudice, 35 (1).

DieHL, C.; Urbahn, J. (1999). Die soziale und politische Partizipation von Zuwanderern in der Bundesrepublik Deutschland [en línea]. <http://library.fes.de/fulltext/ asfo/00258toc.htm> [Consulta: 15 de abril de 2007]

GofFman, E. (1959). The presentation of self in everyday life. Nueva York: Doubleday.

GIUGNI, M.; PASSY, F. (2004). «Migrant mobilization between political institutions and citizenship regimes: A comparison of France and Switzerland». European Journal of Political Research, 43 (1).

GuSTAFSON, P. (2001). «Retirement migration and transnational lifestyles». Ageing and Society, 21 (4).

HarWoOd, S.; Myers, D. (2002). «The dynamics of immigration and local governance in Santa Ana: Neighborhood activism, overcrowding, and land-use policy». Policy Studies Journal, 30 (1).

HeilmanN, C. (2006). «Remittances and the migration-development nexus: Challenges for the sustainable governance of migration». Ecological Economics, 59 (2).

INE. Instituto NaCiOnAl De Estadística. (2000, 2007). Exploración del padrón [en línea]. <http://www.ine.es> [Consulta: 15 de abril de 2007]

Jacobs, D.; Phalet, K.; Swynegedouw, M. (2004). «Associational Membership and Political Involvement Among Ethnic Minority Groups in Brussels». Journals of Ethnic and Migration Studies, vol 30, $\mathrm{n}^{\circ} 3$.

JunN, J. (1999). «Participation in liberal democracy: the political assimilation of immigrants and ethnic minorities in the United States». American Behavioral Scientist, $42(9)$. 
Kitschelt, H. P. (1986). «Political Opportunity Structures and Political Protest: AntiNuclear Movements in Four Democracies». British Journal of Political Science, 16. Koopmans, R. (1998). "Konkurrierende Paradigmen oder friedlich ko-existiernde Komplemente? Eine Bilanz der Theorien sozialer Bewegungen». En: Hellmann, K; U.; Koopmans, R. (eds.). Paradigmen der Bewegungsforschung. Opladen/ Wiesbaden: Westdeutscher Verlag.

KoOpMANS, R. (2004). «Migrant mobilisation and political opportunities: Variation among German cities and a comparison with the United Kingdom and the Netherlands». Journal of Ethnic and Migration Studies, 30 (3).

Kriesi, H.; Koopmans, R.; DuyvendaK, J.; Giugini, M. G. (1992). «New Social Movements and Political Opportunities in Western Europe». European Journal of Political Research, vol. 22.

KYMLICKA, W. (1995). Multicultural citizenship: A liberal theory of minority rights. Oxford: University Press.

DE LUCAS, J. (2004). "Globalización, migraciones y derechos humanos. La inmigración como res política». Cuadernos Electrónicos de Filosofía del Derecho, 10 [en línea]. <http://www.uv.es/CEFD/10/delucas.pdf> [Consulta: 15 de abril de 2007]

Martín PÉrez, A. (2006). «Doing Qualitative Migration Research as a Native Citizen of the Host Country: Reflections on a Research in Spain». Forum: Qualitative Social Research, 7(3) [en línea]. <http://www.qualitative-research.net/fqs-texte/306/06-3-1-e.htm> [Consulta: 15 de abril de 2007]

MCCARTHY, J.; MAYER, Z. (1977). «Resource Mobilization and Social Movements: A Partial Theory». The American Journal of Sociology, vol. 82, $\mathrm{n}^{\circ} 6$.

Melucci, A. (1980). «The New Social Movements: A Theoretical Approach». Social Sciences Information, 19 (2).

Miethe, I.; Roth, S. (eds.) (2000). Politische Biografien und sozialer Wandel. Gießen: Psychosozial-Verlag.

Morales, L.; GONZALES, A.; SÁNCHEZ, G. (2005). «La integración política de los inmigrantes. Un estudio sobre el asociacionismo en Madrid y Murcia». Cuadernos Electrónicos de Filosofía de Derecho, 12 [en línea]. <http://www.uv.es/CEFD/12/ sanchez.pdf> [Consulta: 15 de abril de 2007]

Nentwich, M. (1996). «Opportunity Structures for Citizens' Participation: The Case of the European Union». European Integration Online Papers (EIoP), vol. 0, $\mathrm{n}^{\circ} 1$ [en línea]. <http://eiop.or.at/eiop/texte/1996-001.htm> [Consulta: 15 de abril de 2007]

Odmalm, P. (2004). «Civil Society, Migrant Organisations and Political Parties: Theoretical Linkages and Applications to the Swedish Context». Journals of Ethnic and Migration Studies, vol 30, no 3 .

PARDO, M. (1990). Latinas and urban grassroots activism: Social identities as a mobilization resource. Conference Paper, International Sociological Association (ISA).

Parsons, T. (1959). «Sociological Aspect of Facists Movements», en: Politics and social structure. Nueva York y Londres: Free Press \& Collier-Macmillan.

PETIT, A. (2005). «La participación desde el enfoque de género». Cuadernos Electrónicos de Filosofía del Derecho, no 12 [en línea]. <http://www.uv.es/CEFD/12/petit.pdf> [Consulta: 15 de abril de 2007]

PutNAm, R. (2000). Bowling alone. The collapse and revival of american community. Nova York: Simon \& Schuster.

RAMAKRISHNAN, S. K.; ESPENSHADE, T. J. (2001). «Immigrant incorporation and political participation in the United States». International Migration Review, 35(3). 
SCHrover, M.; Vermeulen, F. (2005). «Immigrant organisations». Journal of Ethnic and Migration Studies, 31 (5).

Simó, C.; JAbBAZ, M.; Torres, F.; Giner, J.; Herzog, B. (2005). «Asociacionismo y población extranjera en la Comunidad Valenciana». Cuadernos Electrónicos de Filosofía del Derecho, no 12 [en línea]. <http://www.uv.es/CEFD/12/simo.pdf> [Consulta: 15 de abril de 2007]

SNOW, D.; BENFord, R. (1992). «Master Frames and Cycles of Protest». En: MorRIS, A.; Mueller, C. (eds.). Frontiers in social movement theory. New Haven: Yale University Press.

TORRES, F. (2005). Ámbit urbà, sociabilitat $i$ inserció social dels immigrants: El cas de Russafa (València). Tesis doctoral dirigida por Na Josepa Cucó i Giner, Valencia. Uehlinger, H.-M. (1988). Politische Partizipation in der Bundesrepublik. Opladen: Westdeutscher Verlag. 